\title{
An Introduction to Constraint Programming and Combinatorial Optimisation
}

\author{
Barry O’Sullivan \\ Cork Constraint Computation Centre, \\ Department of Computer Science, University College Cork, Cork, Ireland \\ b.osullivan@4c.ucc.ie
}

\begin{abstract}
Computers play an increasingly important role in helping individuals and industries make decisions. For example they can help individuals make decisions about which products to purchase or industries make decisions about how best to manufacture these products. Constraint programming provides powerful support for decision-making; it is able to search quickly through an enormous space of choices, and infer the implications of those choices. This tutorial will teach attendees how to develop models of combinatorial problems and solve them using constraint programming, satisfiability and mixed integer programming techniques. The tutorial will make use of Numberjack, an open-source Python-based optimisation system developed at the Cork Constraint Computation Centre. The focus of the tutorial will be on various network design problems and optimisation challenges in the Web.
\end{abstract}

